\title{
Network Resiliency Implementation in the ATLAS TDAQ System
}

\author{
Stefan Stancu
}




\section{Outline}

- TDAQ system block diagram

- Networks and protocols

it Global view

¿ Control network

is Front/Back-End network

- Operational issues

- Conclusions 


\section{TDAQ system block diagram}

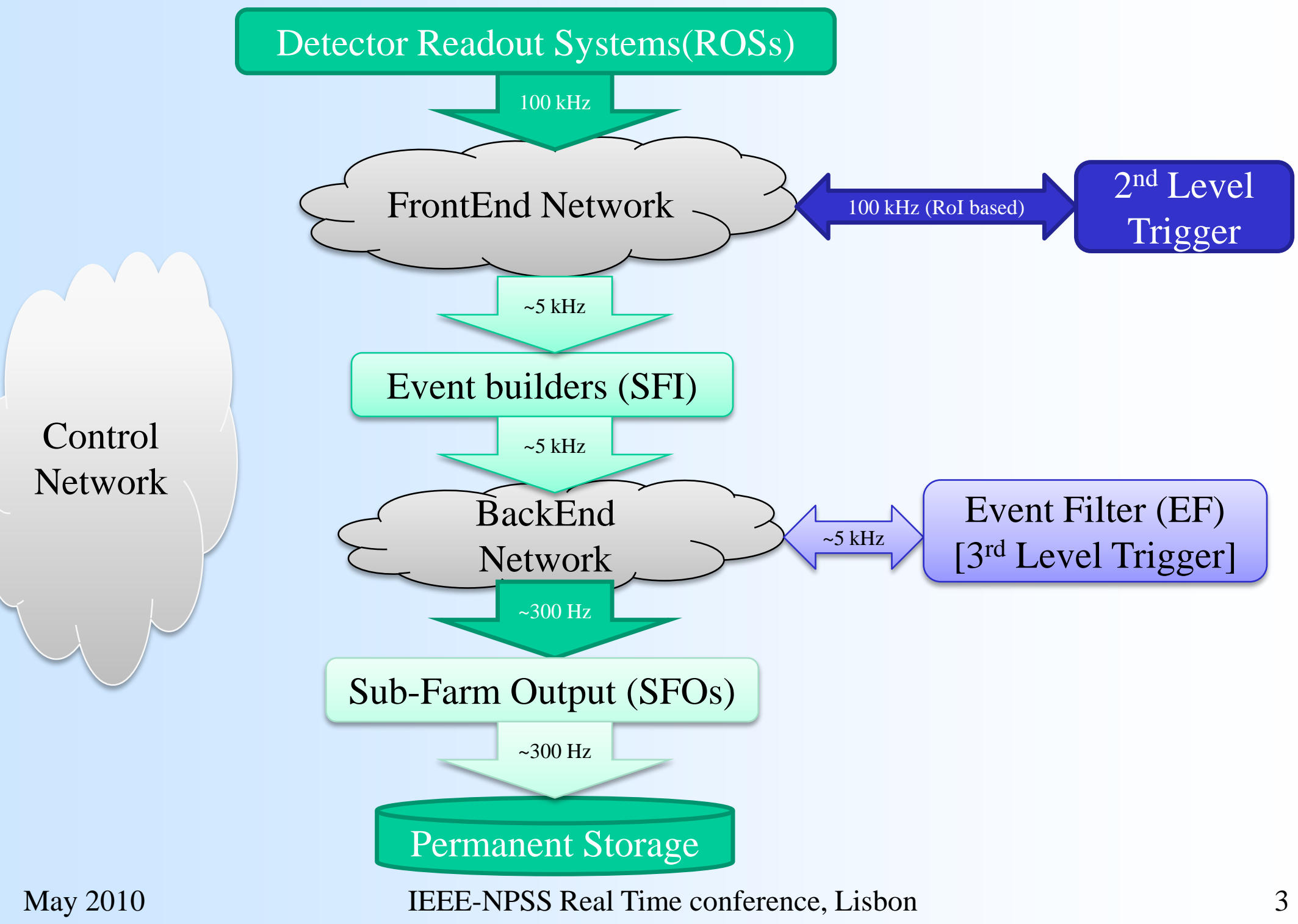




\section{TDAQ system block diagram}

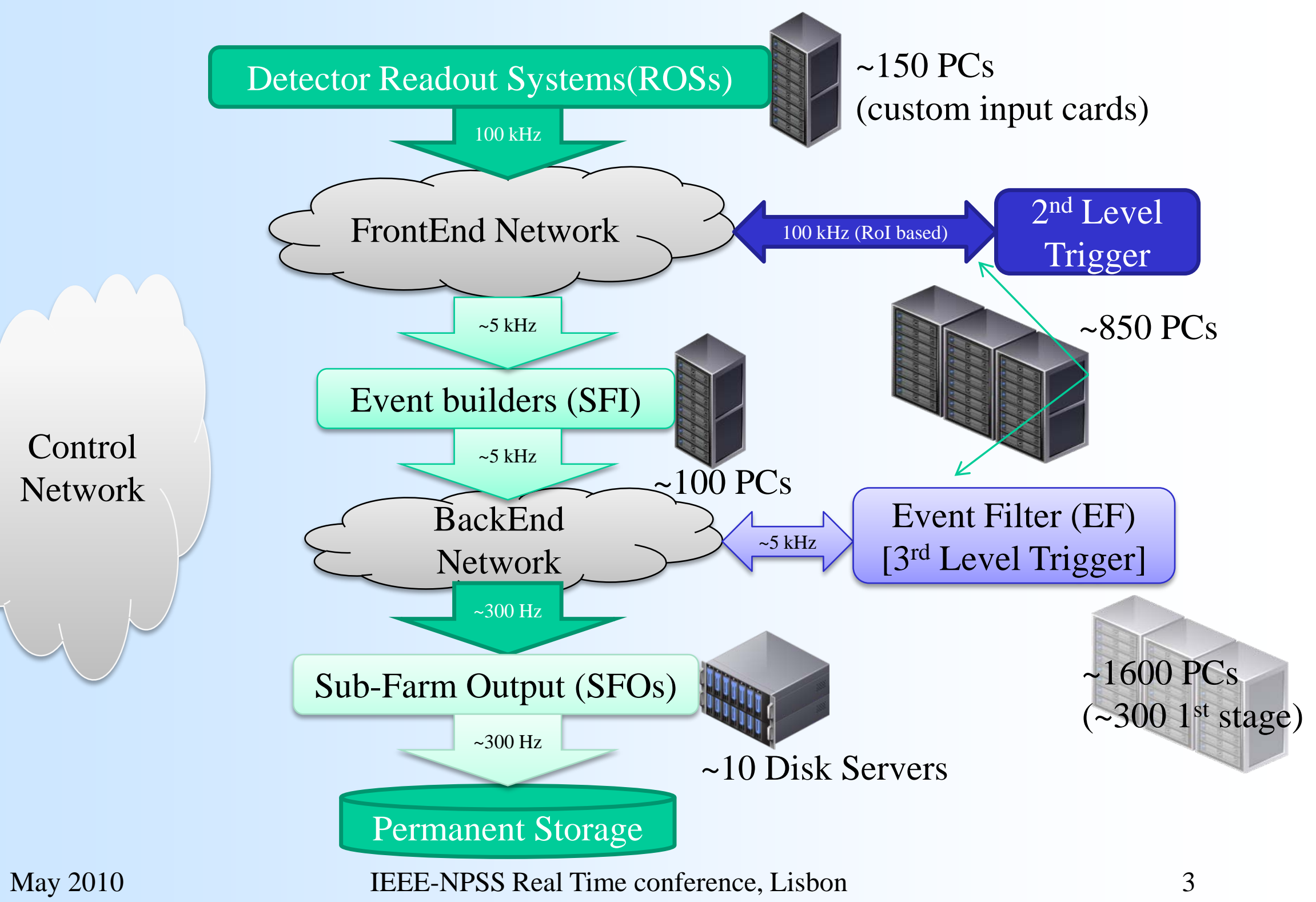




\section{TDAQ system block diagram}

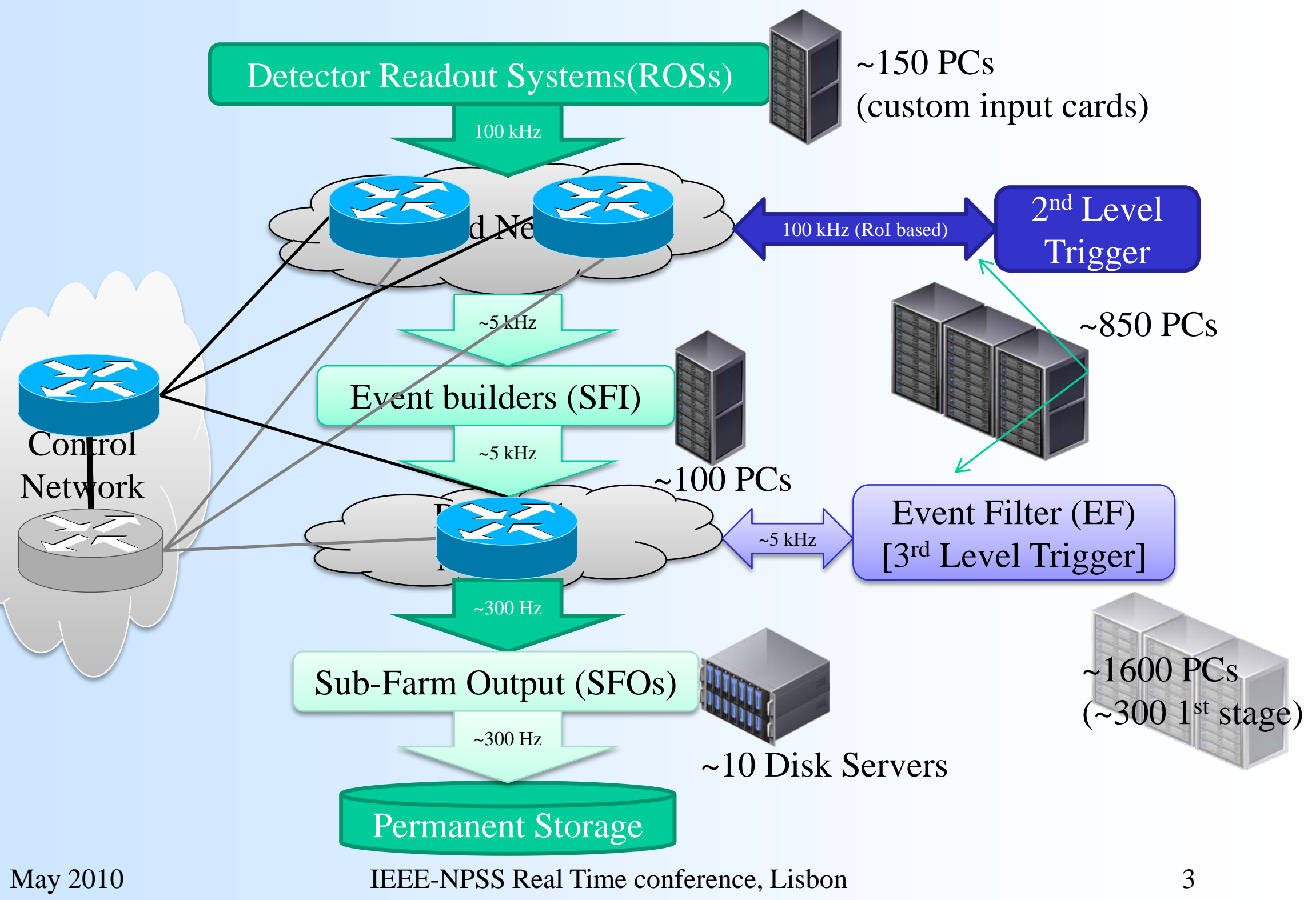




\section{Global view - routers}

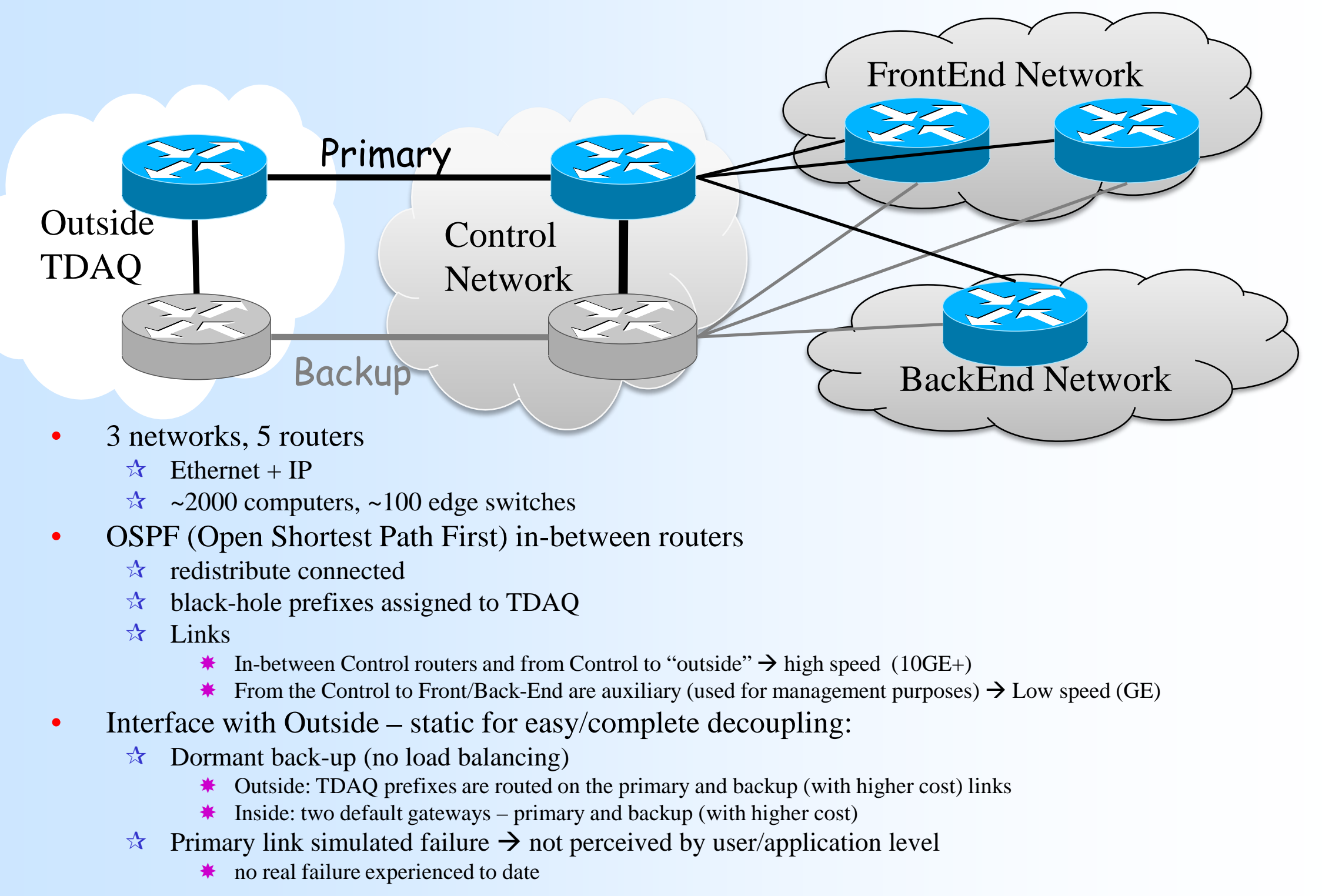

May 2010

IEEE-NPSS Real Time conference, Lisbon 


\section{Control network - VRRP}

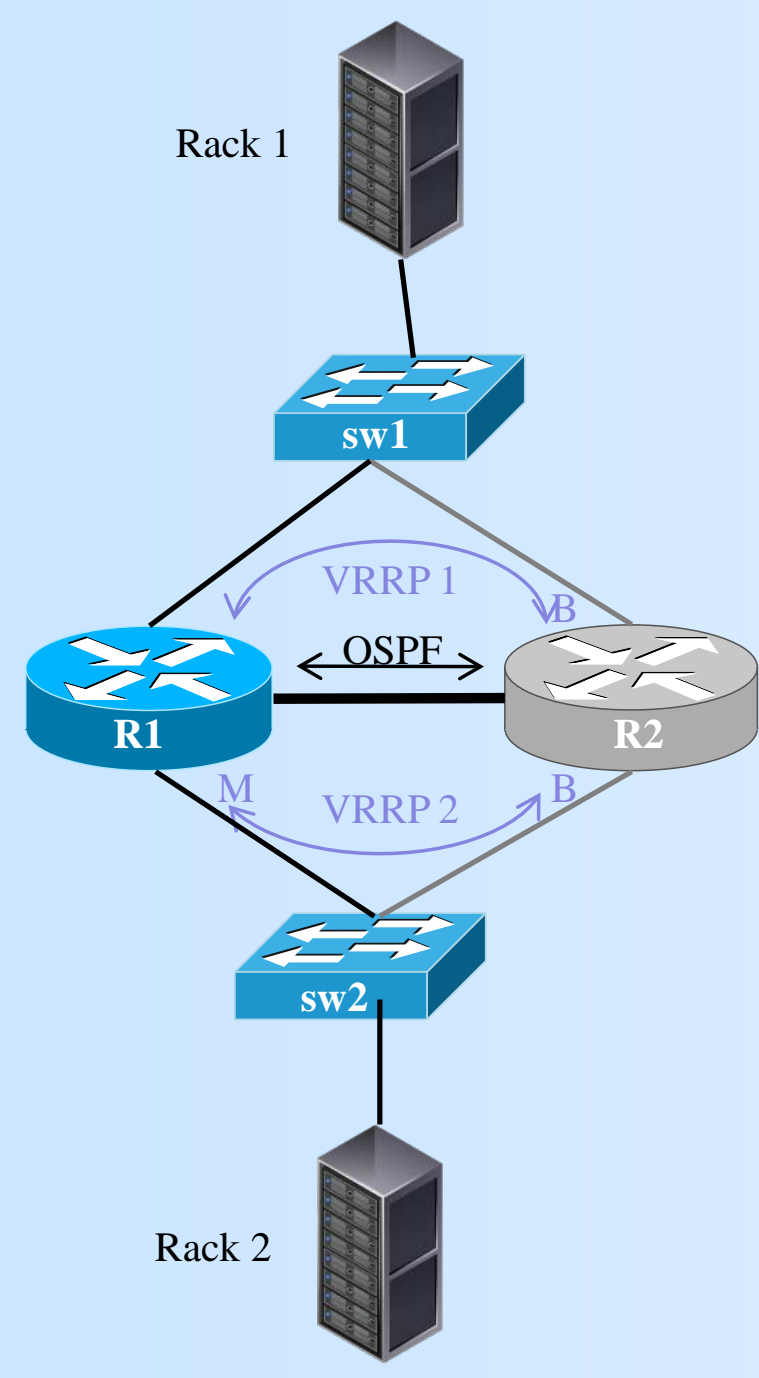

- Inter-router link runs OSPF ( "redistributes connected")

is Two trunked 10GE lines

- R1,R2 provide:

ते subnet 1 to sw1

is subnet 2 to sw2

- VRRP (Virtual Router Redundancy Protocol) operation:

is SubnetX $\rightarrow$ VRRP instance X:

* One MAC (vrrp_macX) and one IP (vrrp_ipX) for the virtual router

* Physical routers hand-shake and elect:

$\bigcirc$ a master router (R1) $\rightarrow$ implements the virtual router

$\bigcirc$ a backup router (R2) $\rightarrow$ dormant while the master is active

is R1-sw2 link fails

* R1-R2 handshake on subnet 2 fails (as R1 is not reachable through sw2)

* R2 no longer sees a master so it becomes the master itself, implementing the virtual router (with vrrp_mac2, and vrrp_ip2)

* Hosts in Rack2 continue to talk to the virtual router, unaware of the physical change

- A single VRRP instance provides redundancy but no load balancing

is Two VRRP instances per subnet (R1 master in one instance, R2 master in the other one) could provide load balancing

* However this causes asymmetric traffic (potential flooding on sw1, sw2)

* To be avoided if bandwidth is not an issue 


\section{Control network - VRRP}

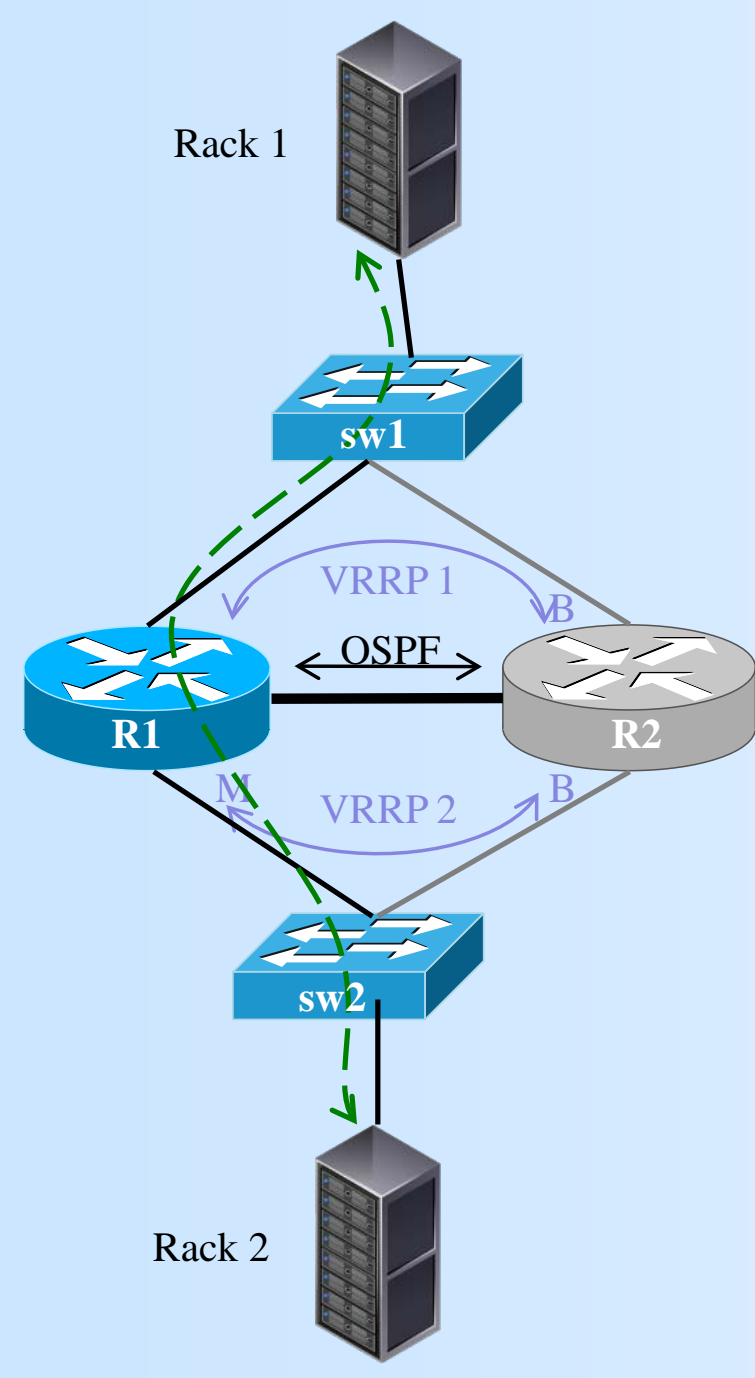

- Inter-router link runs OSPF ( "redistributes connected")

is Two trunked 10GE lines

- R1,R2 provide:

ते subnet 1 to sw1

is subnet 2 to sw2

- VRRP (Virtual Router Redundancy Protocol) operation:

is SubnetX $\rightarrow$ VRRP instance X:

* One MAC (vrrp_macX) and one IP (vrrp_ipX) for the virtual router

* Physical routers hand-shake and elect:

$\bigcirc$ a master router (R1) $\rightarrow$ implements the virtual router

$\bigcirc$ a backup router (R2) $\rightarrow$ dormant while the master is active

is R1-sw2 link fails

* R1-R2 handshake on subnet 2 fails (as R1 is not reachable through sw2)

* R2 no longer sees a master so it becomes the master itself, implementing the virtual router (with vrrp_mac2, and vrrp_ip2)

* Hosts in Rack2 continue to talk to the virtual router, unaware of the physical change

- A single VRRP instance provides redundancy but no load balancing

is Two VRRP instances per subnet (R1 master in one instance, R2 master in the other one) could provide load balancing

* However this causes asymmetric traffic (potential flooding on sw1, sw2)

* To be avoided if bandwidth is not an issue 


\section{Control network - VRRP}

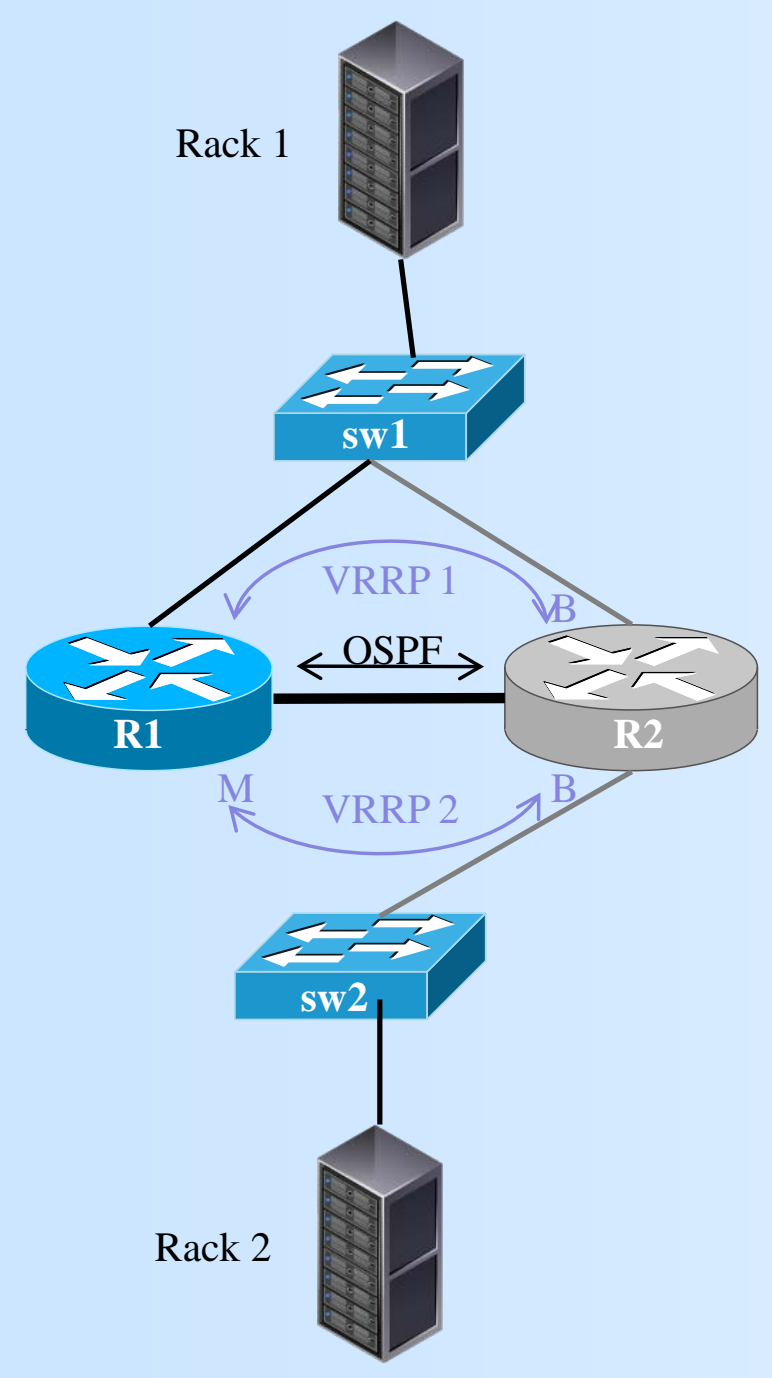

- Inter-router link runs OSPF ( "redistributes connected")

is Two trunked 10GE lines

- R1,R2 provide:

ते subnet 1 to sw1

is subnet 2 to sw2

- VRRP (Virtual Router Redundancy Protocol) operation:

is SubnetX $\rightarrow$ VRRP instance X:

* One MAC (vrrp_macX) and one IP (vrrp_ipX) for the virtual router

* Physical routers hand-shake and elect:

$\bigcirc$ a master router (R1) $\rightarrow$ implements the virtual router

$\bigcirc$ a backup router (R2) $\rightarrow$ dormant while the master is active

is R1-sw2 link fails

* R1-R2 handshake on subnet 2 fails (as R1 is not reachable through sw2)

* R2 no longer sees a master so it becomes the master itself, implementing the virtual router (with vrrp_mac2, and vrrp_ip2)

* Hosts in Rack2 continue to talk to the virtual router, unaware of the physical change

- A single VRRP instance provides redundancy but no load balancing

is Two VRRP instances per subnet (R1 master in one instance, R2 master in the other one) could provide load balancing

* However this causes asymmetric traffic (potential flooding on sw1, sw2)

* To be avoided if bandwidth is not an issue 


\section{Control network - VRRP}

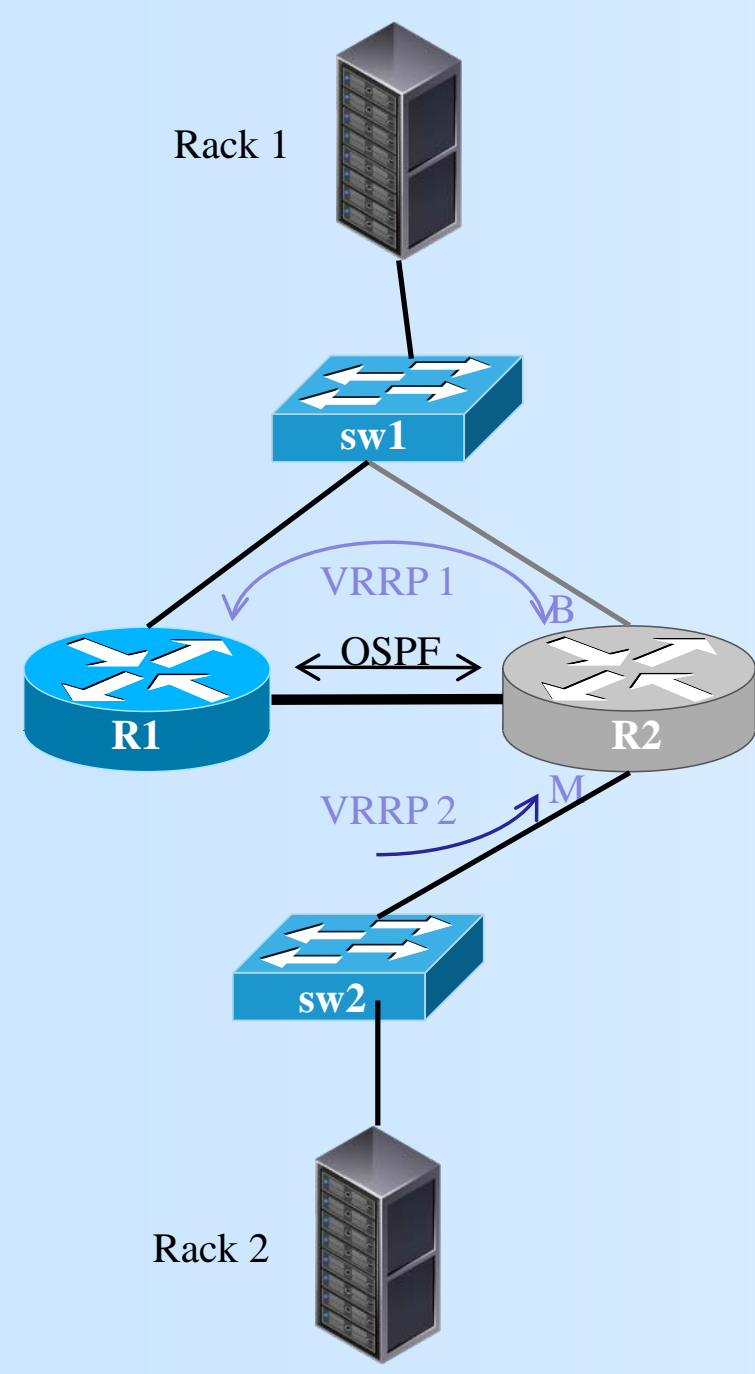

- Inter-router link runs OSPF ( "redistributes connected")

is Two trunked 10GE lines

- R1,R2 provide:

ते subnet 1 to sw1

is subnet 2 to sw2

- VRRP (Virtual Router Redundancy Protocol) operation:

is SubnetX $\rightarrow$ VRRP instance X:

* One MAC (vrrp_macX) and one IP (vrrp_ipX) for the virtual router

* Physical routers hand-shake and elect:

$\bigcirc$ a master router (R1) $\rightarrow$ implements the virtual router

$\bigcirc$ a backup router (R2) $\rightarrow$ dormant while the master is active

is R1-sw2 link fails

* R1-R2 handshake on subnet 2 fails (as R1 is not reachable through sw2)

* R2 no longer sees a master so it becomes the master itself, implementing the virtual router (with vrrp_mac2, and vrrp_ip2)

* Hosts in Rack2 continue to talk to the virtual router, unaware of the physical change

- A single VRRP instance provides redundancy but no load balancing

is Two VRRP instances per subnet (R1 master in one instance, R2 master in the other one) could provide load balancing

* However this causes asymmetric traffic (potential flooding on sw1, sw2)

* To be avoided if bandwidth is not an issue 


\section{Control network - VRRP}

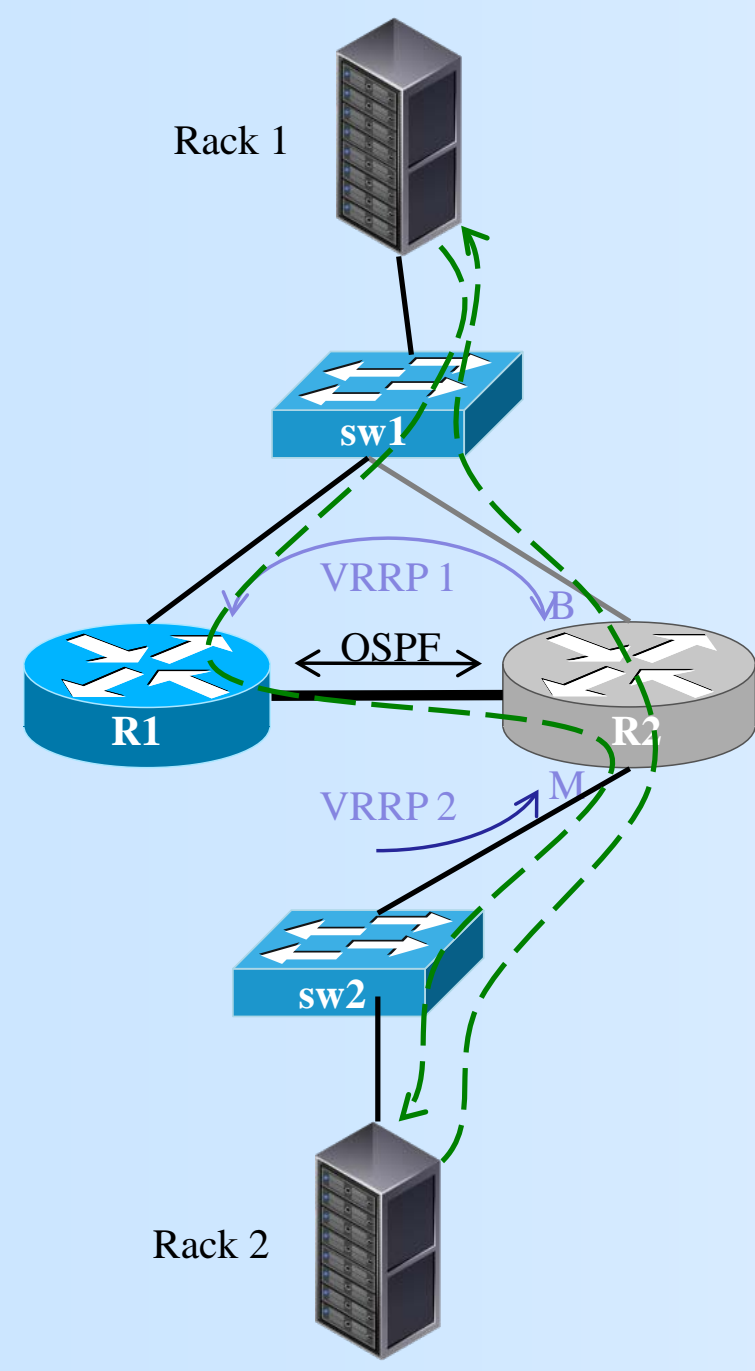

- Inter-router link runs OSPF ( "redistributes connected")

is Two trunked 10GE lines

- R1,R2 provide:

is subnet 1 to sw1

is subnet 2 to sw2

- VRRP (Virtual Router Redundancy Protocol) operation:

is SubnetX $\rightarrow$ VRRP instance X:

* One MAC (vrrp_macX) and one IP (vrrp_ipX) for the virtual router

* Physical routers hand-shake and elect:

$\bigcirc$ a master router (R1) $\rightarrow$ implements the virtual router

$\bigcirc$ a backup router (R2) $\rightarrow$ dormant while the master is active

is R1-sw2 link fails

* R1-R2 handshake on subnet 2 fails (as R1 is not reachable through sw2)

* R2 no longer sees a master so it becomes the master itself, implementing the virtual router (with vrrp_mac2, and vrrp_ip2)

* Hosts in Rack2 continue to talk to the virtual router, unaware of the physical change

- A single VRRP instance provides redundancy but no load balancing

is Two VRRP instances per subnet (R1 master in one instance, R2 master in the other one) could provide load balancing

* However this causes asymmetric traffic (potential flooding on sw1, sw2)

* To be avoided if bandwidth is not an issue 


\section{Control network - VRRP}

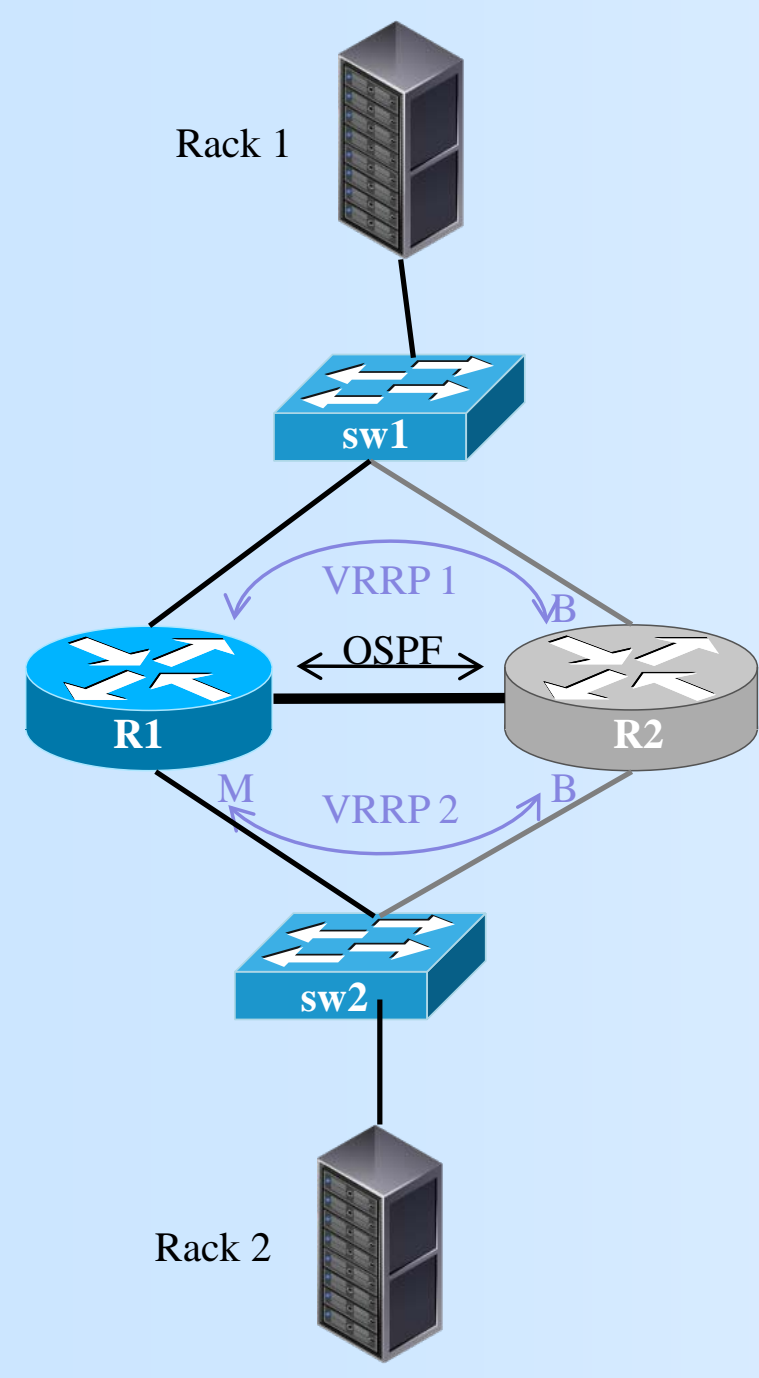

- Inter-router link runs OSPF ( "redistributes connected")

is Two trunked 10GE lines

- R1,R2 provide:

ते subnet 1 to sw1

is subnet 2 to sw2

- VRRP (Virtual Router Redundancy Protocol) operation:

is SubnetX $\rightarrow$ VRRP instance X:

* One MAC (vrrp_macX) and one IP (vrrp_ipX) for the virtual router

* Physical routers hand-shake and elect:

$\bigcirc$ a master router (R1) $\rightarrow$ implements the virtual router

$\bigcirc$ a backup router (R2) $\rightarrow$ dormant while the master is active

is R1-sw2 link fails

* R1-R2 handshake on subnet 2 fails (as R1 is not reachable through sw2)

* R2 no longer sees a master so it becomes the master itself, implementing the virtual router (with vrrp_mac2, and vrrp_ip2)

* Hosts in Rack2 continue to talk to the virtual router, unaware of the physical change

- A single VRRP instance provides redundancy but no load balancing

is Two VRRP instances per subnet (R1 master in one instance, R2 master in the other one) could provide load balancing

* However this causes asymmetric traffic (potential flooding on sw1, sw2)

* To be avoided if bandwidth is not an issue 


\section{Control network - VRRP}

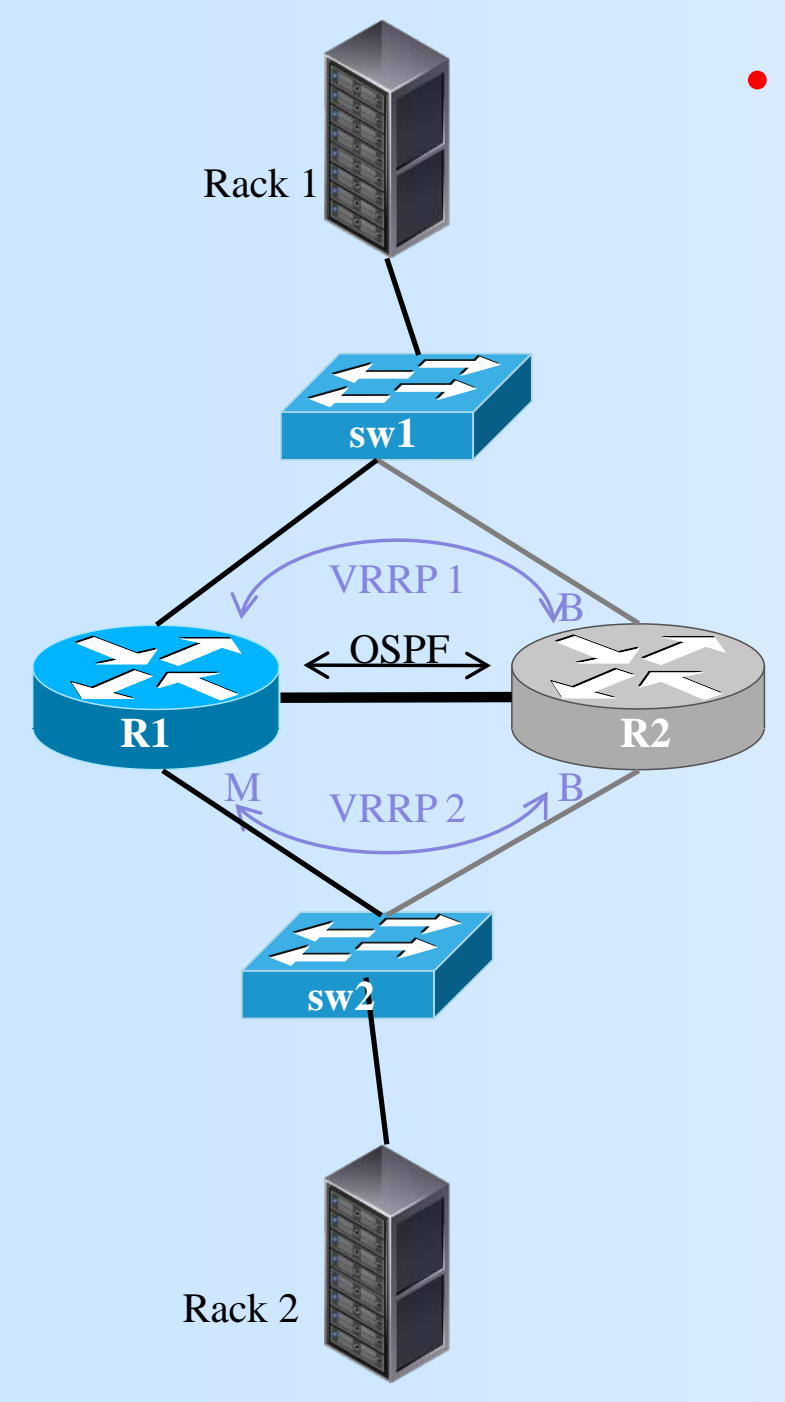

- Practical issues

is Tested prior to deployment

is With proxy ARP enabled the following happens when host a host from rack 2 (host_r2) wants to talk outside subnet 2:

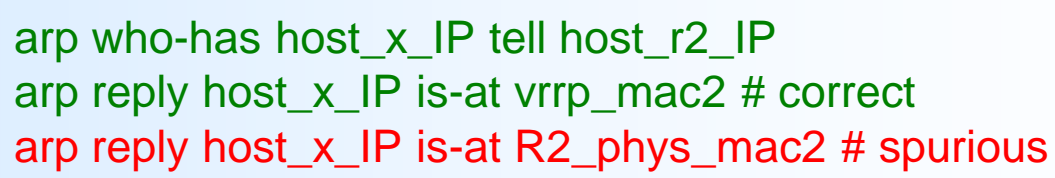

is Depending on which ARP reply is received first (can be assumed to be random), the host will

* either behave correctly (correct ARP received first)

* or will use the "backup" router R2 (spurious ARP received first)

is Undesired behaviour because:

* Traffic through R2 is asymmetric (return comes through R1) flooding can occur on sw2 depending on its mac-address-aging settings

* Uncontrolled load balancing

* Will not be detected on a test which only disables one swX primary link to R1

$\dot{*}$ Deployed in production only after the issue was fixed by the manufacturer

- If possible thoroughly test before deployment. 


\section{Control network - VRRP}

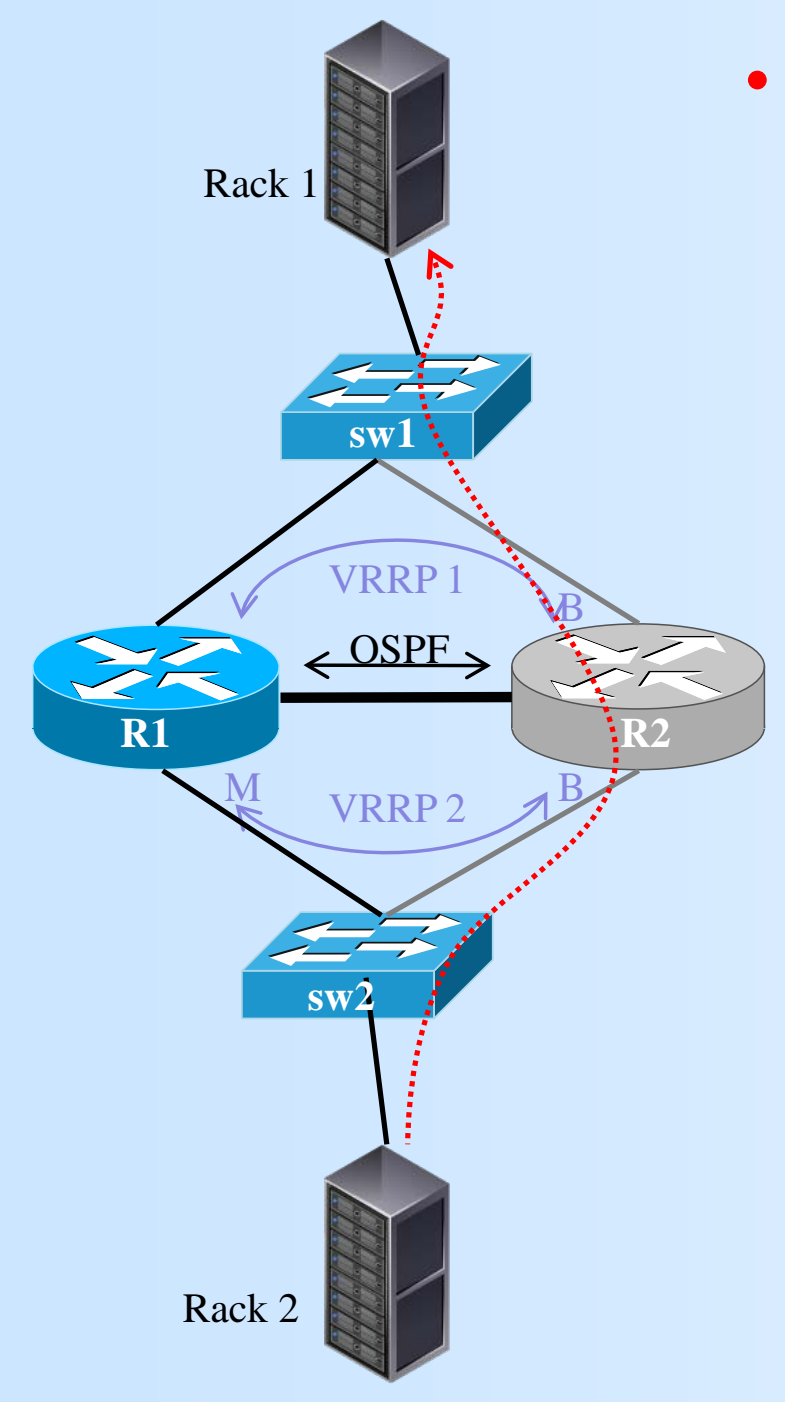

- Practical issues

is Tested prior to deployment

is With proxy ARP enabled the following happens when host a host from rack 2 (host_r2) wants to talk outside subnet 2:

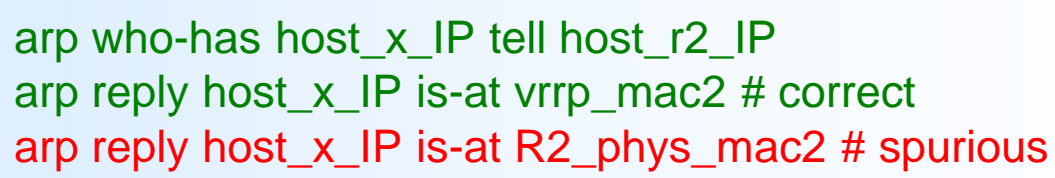

is Depending on which ARP reply is received first (can be assumed to be random), the host will

* either behave correctly (correct ARP received first)

* or will use the "backup" router R2 (spurious ARP received first)

is Undesired behaviour because:

* Traffic through R2 is asymmetric (return comes through R1) flooding can occur on sw2 depending on its mac-address-aging settings

* Uncontrolled load balancing

* Will not be detected on a test which only disables one swX primary link to R1

$\dot{*}$ Deployed in production only after the issue was fixed by the manufacturer

- If possible thoroughly test before deployment. 


\section{Control network - VRRP}

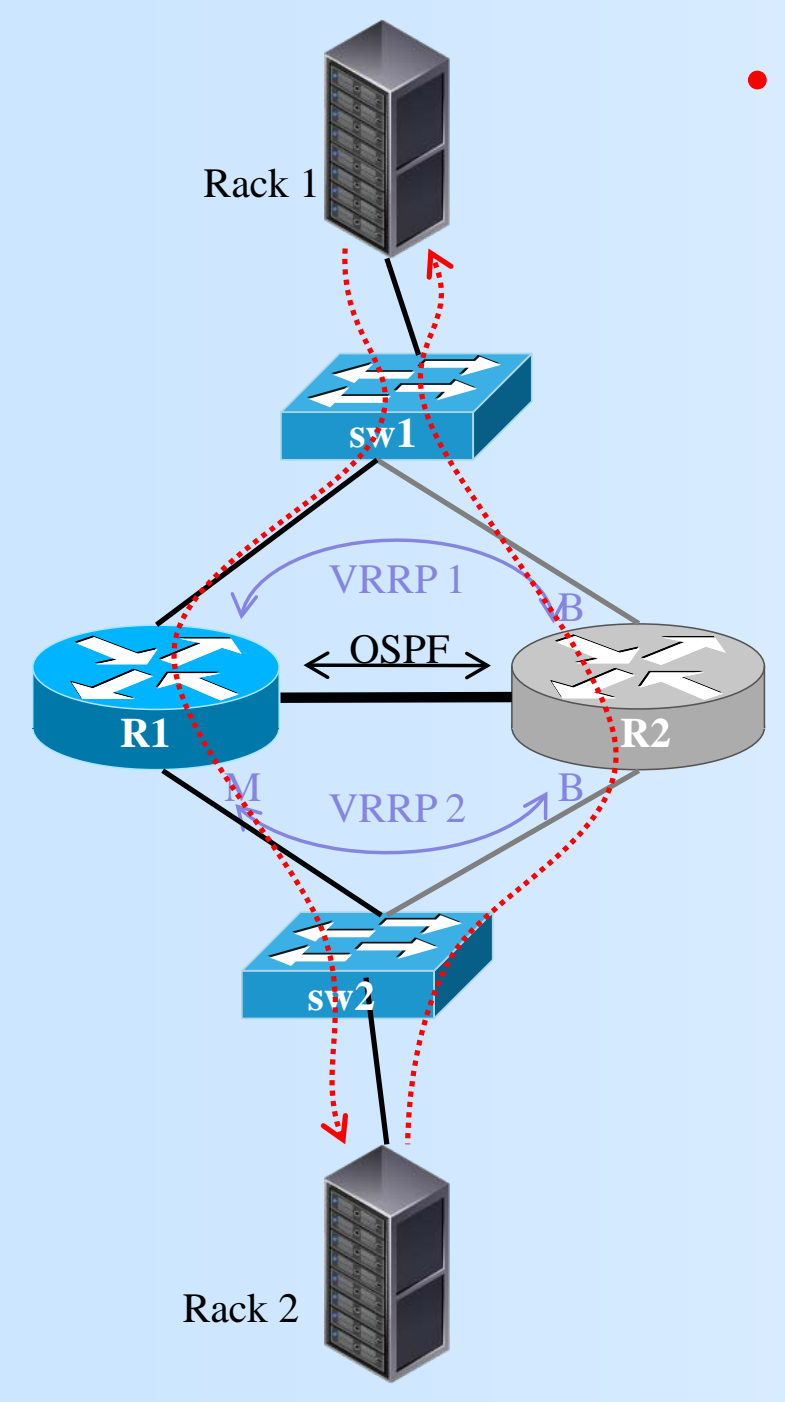

- Practical issues

is Tested prior to deployment

is With proxy ARP enabled the following happens when host a host from rack 2 (host_r2) wants to talk outside subnet 2:

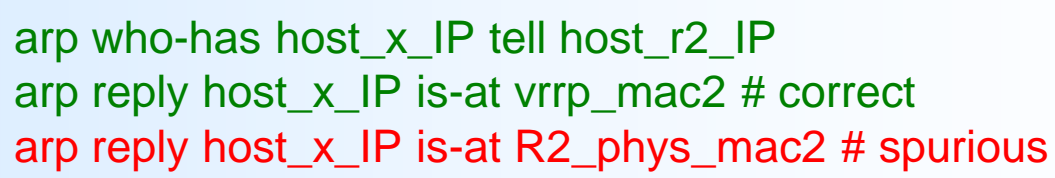

is Depending on which ARP reply is received first (can be assumed to be random), the host will

* either behave correctly (correct ARP received first)

* or will use the "backup" router R2 (spurious ARP received first)

is Undesired behaviour because:

* Traffic through R2 is asymmetric (return comes through R1) flooding can occur on sw2 depending on its mac-address-aging settings

* Uncontrolled load balancing

* Will not be detected on a test which only disables one swX primary link to R1

$\dot{*}$ Deployed in production only after the issue was fixed by the manufacturer

- If possible thoroughly test before deployment. 


\section{Control network -high throughput servers}

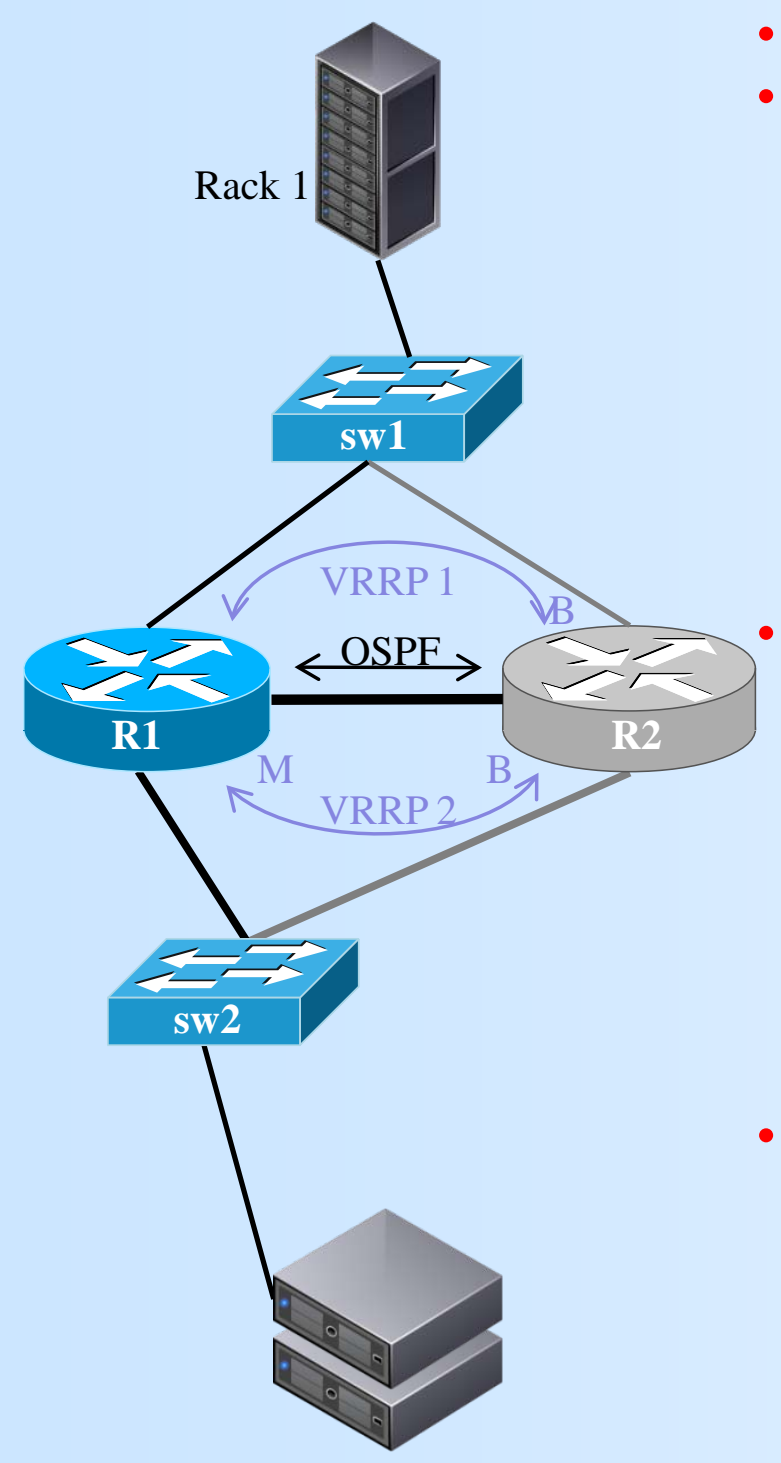

- $\quad$ High throughput needed on 70 infrastructure and monitoring servers

- "Standard” options:

is Edge switch and VRRP with 10G up-links

* Two points of failure (switch and server interface)

is Edge switch and VRRP with 10G up-links + bonding on the server

* Linux bonding in 'active-backup' on the server can provide good redundancy

* One point of failure (switch)

is Two edge switch and VRRP with 10G up-links + bonding on the server

* Linux bonding in 'active-backup' on the server can provide good redundancy

* No single point of failure

* NOTE: STP is required in the subnet in order to break the loops created by the two switches (each one with 2 up-links)

\section{Direct router connections}

is Linux bonding in "active-backup": primary link connected to R1, back-up one to R2

is Not enough:

* example: failure of sw1 primary up-link renders the servers unreachable for Rack1

is Use VLAN interfaces on the routers and interconnect them (emulate a rack level switch)

* the already existing high speed trunk used by OSPF can be shared by virtually any number of VLANs (tagged)

- Production experience

is Deployed for:

* all (most) critical servers

* the NetApp FAS3100 storage units used system wide (user accounts, etc)

is Sample failure while running:

* a server interface going down and then re-negotiation to a lower speed

* no effect perceived on the data taking run

* Shifter reported the warnings generated by the network monitoring tools

May 2010

IEEE-NPSS Real Time conference, Lisbon 


\section{Control network -high throughput servers}

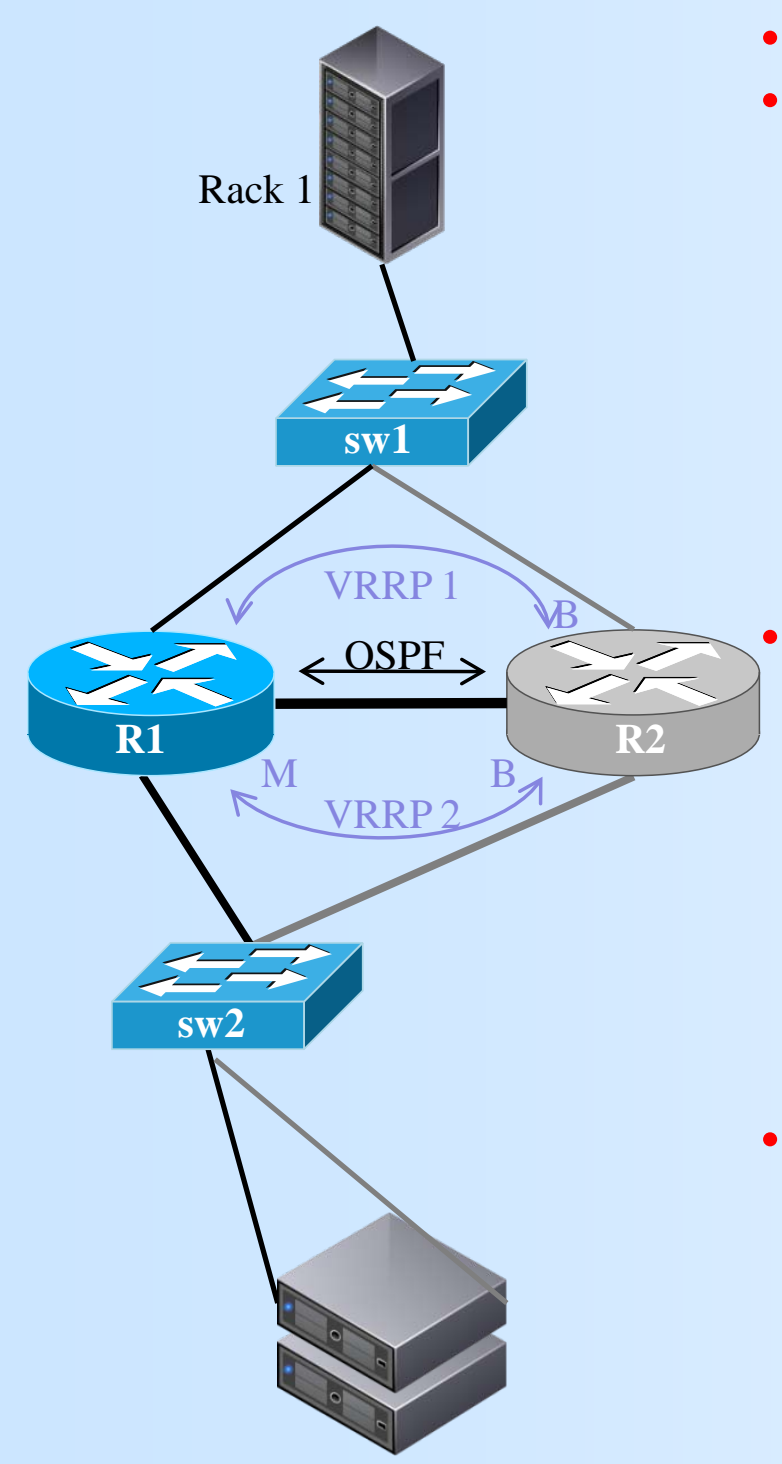

- $\quad$ High throughput needed on 70 infrastructure and monitoring servers

- "Standard” options:

« Edge switch and VRRP with 10G up-links

* Two points of failure (switch and server interface)

is Edge switch and VRRP with 10G up-links + bonding on the server

* Linux bonding in 'active-backup' on the server can provide good redundancy

* One point of failure (switch)

is Two edge switch and VRRP with 10G up-links + bonding on the server

* Linux bonding in ‘active-backup' on the server can provide good redundancy

* No single point of failure

* NOTE: STP is required in the subnet in order to break the loops created by the two switches (each one with 2 up-links)

\section{Direct router connections}

is Linux bonding in "active-backup": primary link connected to R1, back-up one to R2

is Not enough:

* example: failure of sw1 primary up-link renders the servers unreachable for Rack1

is Use VLAN interfaces on the routers and interconnect them (emulate a rack level switch)

* the already existing high speed trunk used by OSPF can be shared by virtually any number of VLANs (tagged)

- Production experience

is Deployed for:

* all (most) critical servers

* the NetApp FAS3100 storage units used system wide (user accounts, etc)

is Sample failure while running:

* a server interface going down and then re-negotiation to a lower speed

* no effect perceived on the data taking run

* Shifter reported the warnings generated by the network monitoring tools

May 2010

IEEE-NPSS Real Time conference, Lisbon 


\section{Control network -high throughput servers}

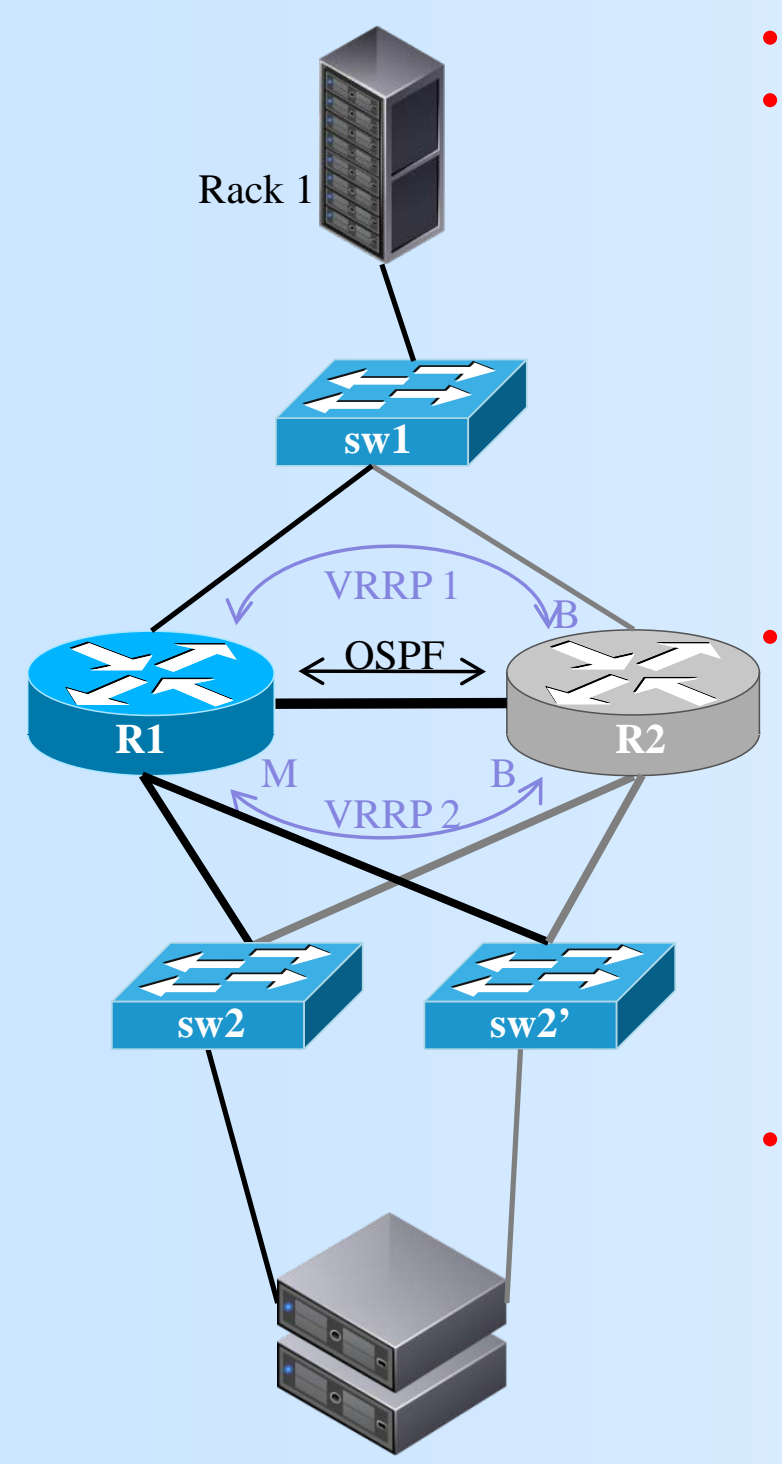

- $\quad$ High throughput needed on 70 infrastructure and monitoring servers

- "Standard” options:

« Edge switch and VRRP with 10G up-links

* Two points of failure (switch and server interface)

is Edge switch and VRRP with 10G up-links + bonding on the server

* Linux bonding in 'active-backup' on the server can provide good redundancy

* One point of failure (switch)

is Two edge switch and VRRP with 10G up-links + bonding on the server

* Linux bonding in 'active-backup' on the server can provide good redundancy

* No single point of failure

* NOTE: STP is required in the subnet in order to break the loops created by the two switches (each one with 2 up-links)

\section{Direct router connections}

is Linux bonding in "active-backup": primary link connected to R1, back-up one to R2

is Not enough:

* example: failure of sw1 primary up-link renders the servers unreachable for Rack1

is Use VLAN interfaces on the routers and interconnect them (emulate a rack level switch)

* the already existing high speed trunk used by OSPF can be shared by virtually any number of VLANs (tagged)

- Production experience

is Deployed for:

* all (most) critical servers

* the NetApp FAS3100 storage units used system wide (user accounts, etc)

is Sample failure while running:

* a server interface going down and then re-negotiation to a lower speed

* no effect perceived on the data taking run

* Shifter reported the warnings generated by the network monitoring tools

May 2010

IEEE-NPSS Real Time conference, Lisbon 


\section{Control network -high throughput servers}

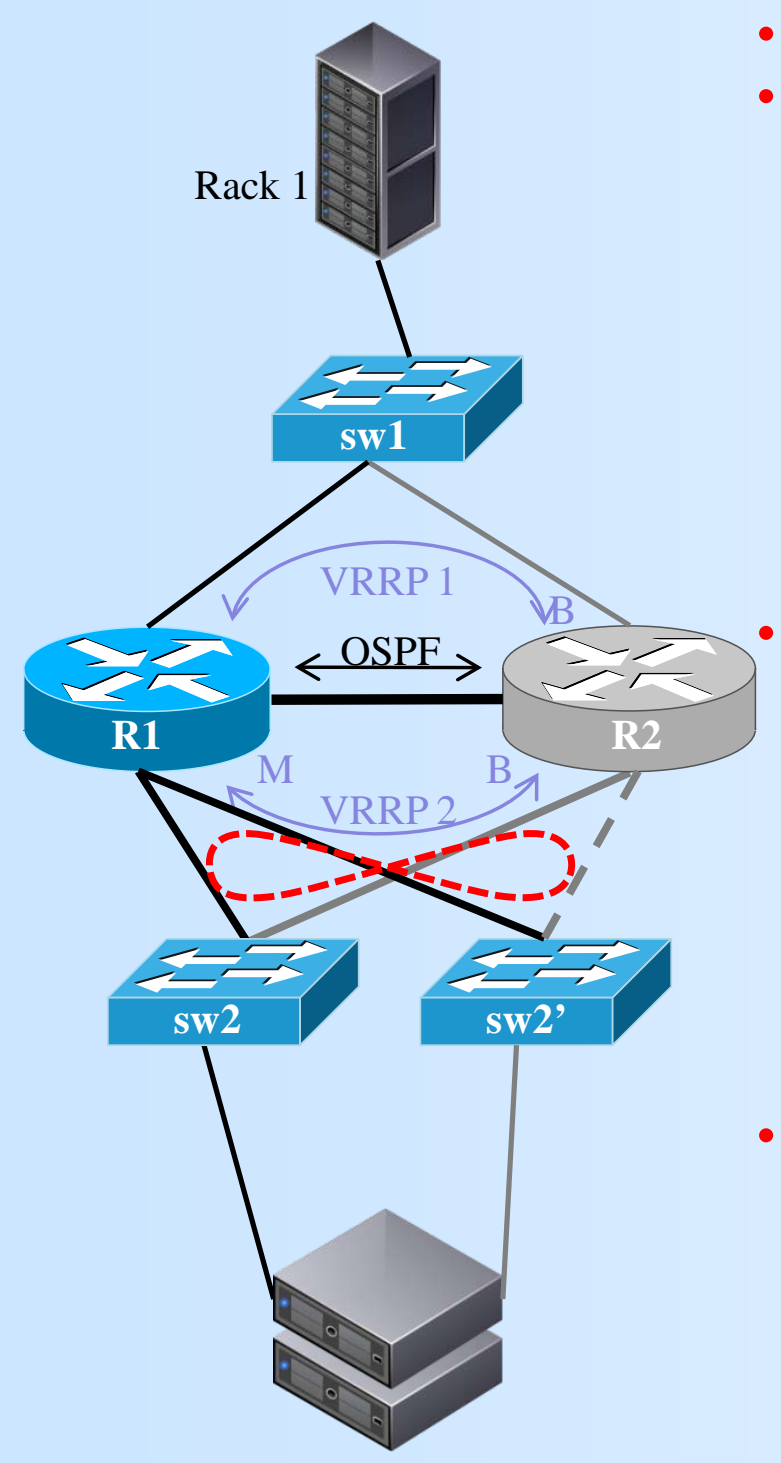

- $\quad$ High throughput needed on 70 infrastructure and monitoring servers

- "Standard” options:

« Edge switch and VRRP with 10G up-links

* Two points of failure (switch and server interface)

is Edge switch and VRRP with 10G up-links + bonding on the server

* Linux bonding in 'active-backup' on the server can provide good redundancy

* One point of failure (switch)

is Two edge switch and VRRP with 10G up-links + bonding on the server

* Linux bonding in 'active-backup' on the server can provide good redundancy

* No single point of failure

* NOTE: STP is required in the subnet in order to break the loops created by the two switches (each one with 2 up-links)

Direct router connections

is Linux bonding in "active-backup": primary link connected to R1, back-up one to R2

is Not enough:

* example: failure of sw1 primary up-link renders the servers unreachable for Rack1

is Use VLAN interfaces on the routers and interconnect them (emulate a rack level switch)

* the already existing high speed trunk used by OSPF can be shared by virtually any number of VLANs (tagged)

- Production experience

is Deployed for:

* all (most) critical servers

* the NetApp FAS3100 storage units used system wide (user accounts, etc)

is Sample failure while running:

* a server interface going down and then re-negotiation to a lower speed

* no effect perceived on the data taking run

* Shifter reported the warnings generated by the network monitoring tools

May 2010

IEEE-NPSS Real Time conference, Lisbon 


\section{Control network -high throughput servers}

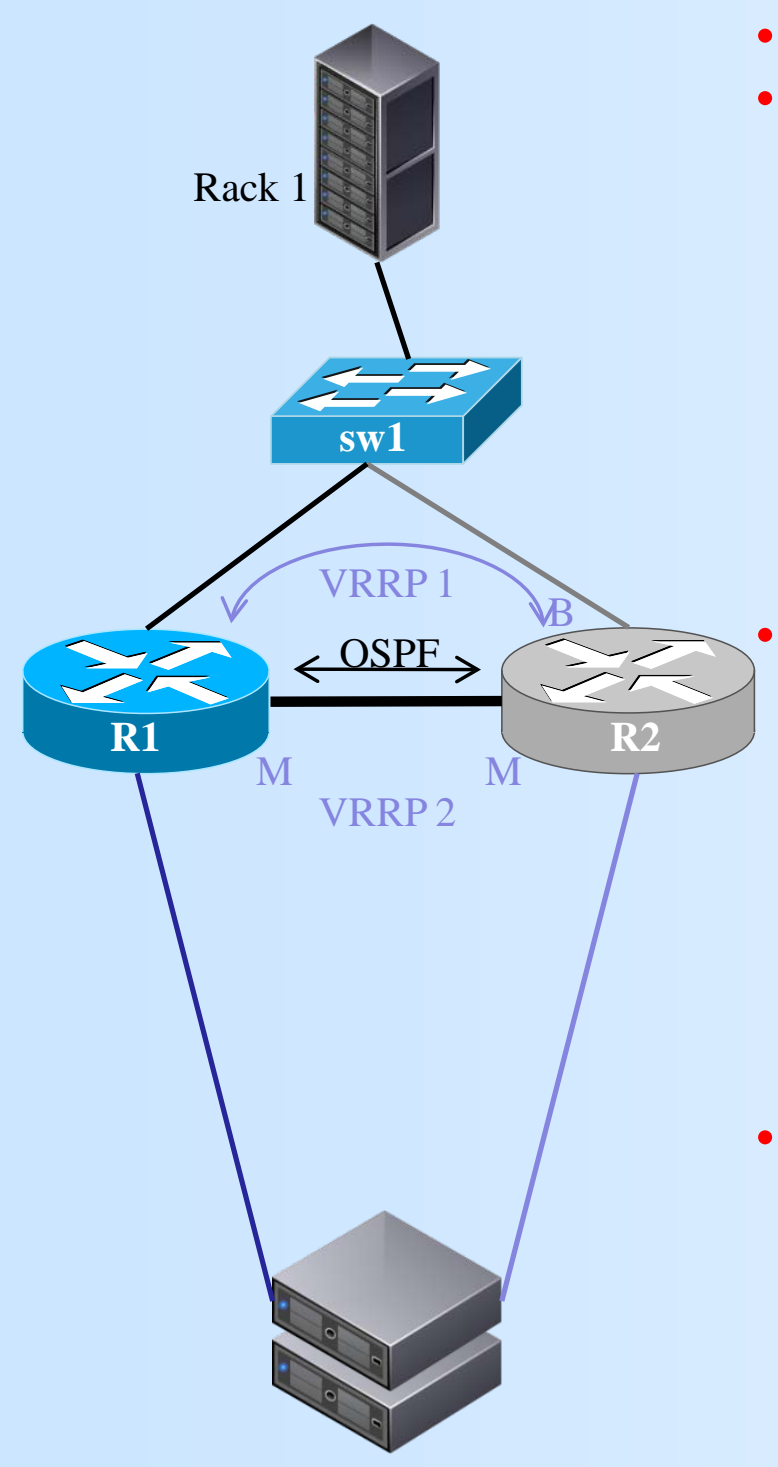

May 2010

- High throughput needed on $~ 70$ infrastructure and monitoring servers

- “Standard” options:

is Edge switch and VRRP with 10G up-links

* Two points of failure (switch and server interface)

is Edge switch and VRRP with 10G up-links + bonding on the server

* Linux bonding in 'active-backup' on the server can provide good redundancy

* One point of failure (switch)

is Two edge switch and VRRP with 10G up-links + bonding on the server

* Linux bonding in 'active-backup' on the server can provide good redundancy

* No single point of failure

* NOTE: STP is required in the subnet in order to break the loops created by the two switches (each one with 2 up-links)

\section{Direct router connections}

is Linux bonding in "active-backup": primary link connected to R1, back-up one to R2

is Not enough:

* example: failure of sw1 primary up-link renders the servers unreachable for Rack1

is Use VLAN interfaces on the routers and interconnect them (emulate a rack level switch)

* the already existing high speed trunk used by OSPF can be shared by virtually any number of VLANs (tagged)

- Production experience

is Deployed for:

* all (most) critical servers

* the NetApp FAS3100 storage units used system wide (user accounts, etc)

is Sample failure while running:

* a server interface going down and then re-negotiation to a lower speed

* no effect perceived on the data taking run

* Shifter reported the warnings generated by the network monitoring tools

IEEE-NPSS Real Time conference, Lisbon 


\section{Control network -high throughput servers}

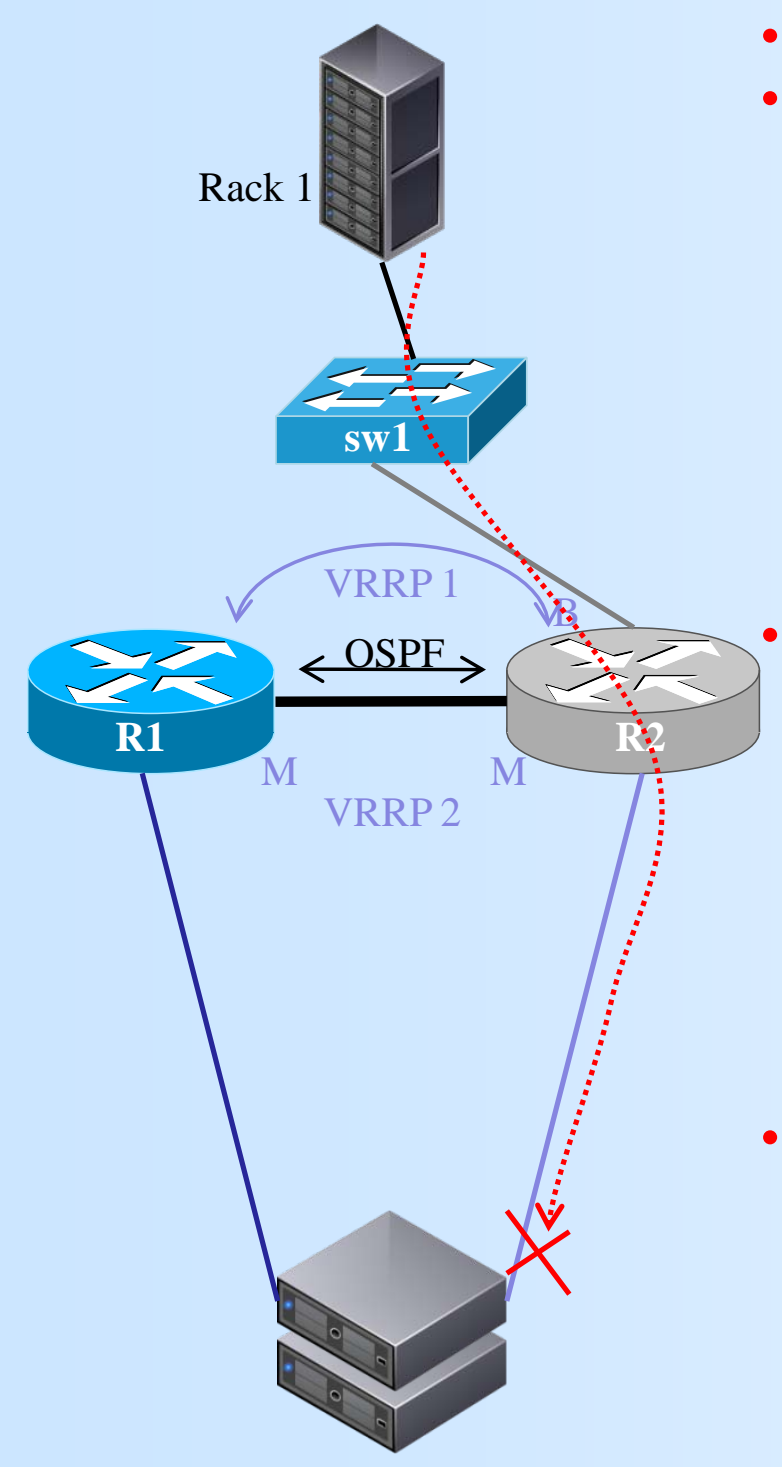

- $\quad$ High throughput needed on 70 infrastructure and monitoring servers

- "Standard” options:

« Edge switch and VRRP with 10G up-links

* Two points of failure (switch and server interface)

is Edge switch and VRRP with 10G up-links + bonding on the server

* Linux bonding in 'active-backup' on the server can provide good redundancy

* One point of failure (switch)

is Two edge switch and VRRP with 10G up-links + bonding on the server

* Linux bonding in 'active-backup' on the server can provide good redundancy

* No single point of failure

* NOTE: STP is required in the subnet in order to break the loops created by the two switches (each one with 2 up-links)

\section{Direct router connections}

is Linux bonding in "active-backup": primary link connected to R1, back-up one to $\mathbf{R} 2$

it Not enough:

* example: failure of sw1 primary up-link renders the servers unreachable for Rack1

is Use VLAN interfaces on the routers and interconnect them (emulate a rack level switch)

* the already existing high speed trunk used by OSPF can be shared by virtually any number of VLANs (tagged)

- Production experience

is Deployed for:

* all (most) critical servers

* the NetApp FAS3100 storage units used system wide (user accounts, etc)

is Sample failure while running:

* a server interface going down and then re-negotiation to a lower speed

* no effect perceived on the data taking run

* Shifter reported the warnings generated by the network monitoring tools

May 2010

IEEE-NPSS Real Time conference, Lisbon 


\section{Control network -high throughput servers}

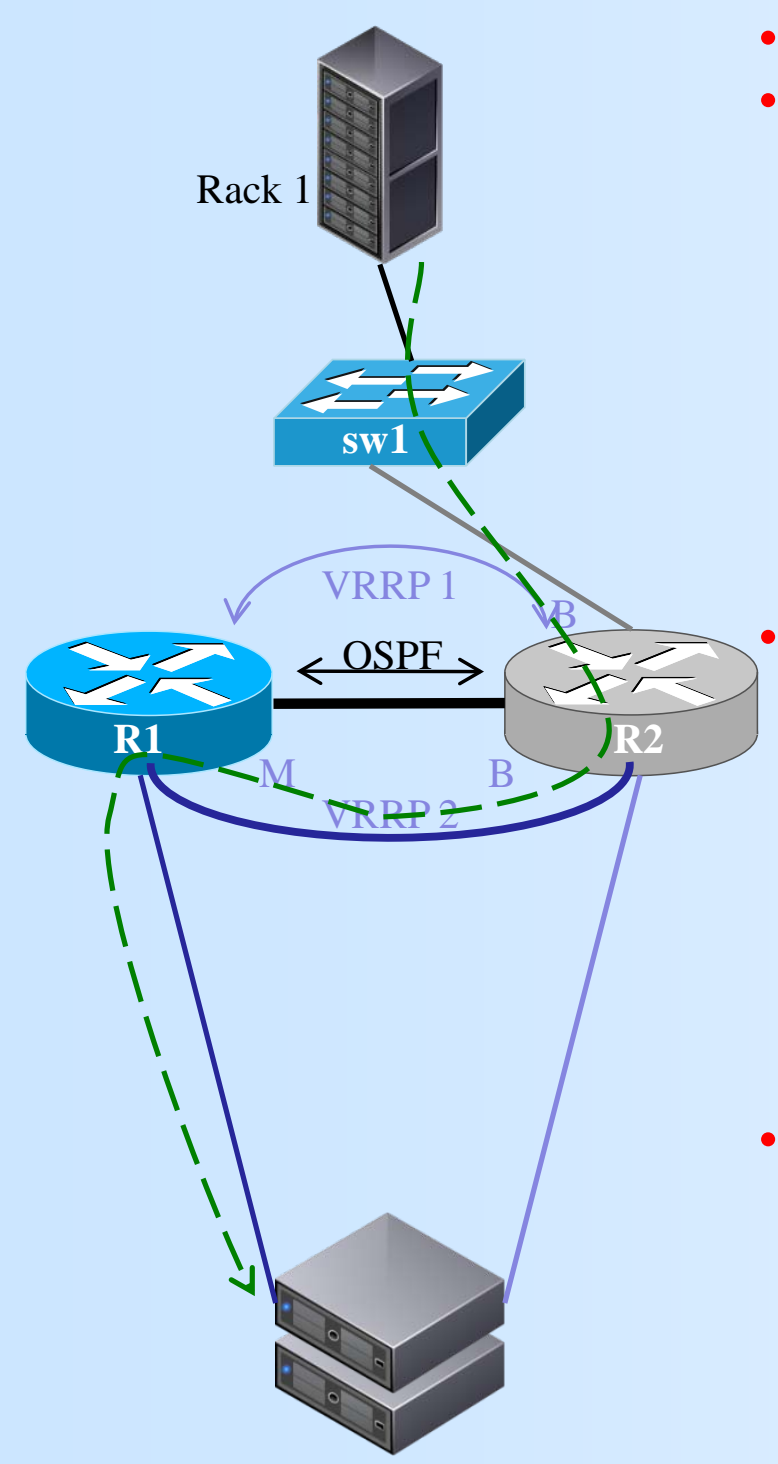

- $\quad$ High throughput needed on $~ 70$ infrastructure and monitoring servers

- "Standard” options:

« Edge switch and VRRP with 10G up-links

* Two points of failure (switch and server interface)

is Edge switch and VRRP with 10G up-links + bonding on the server

* Linux bonding in 'active-backup' on the server can provide good redundancy

* One point of failure (switch)

is Two edge switch and VRRP with 10G up-links + bonding on the server

* Linux bonding in 'active-backup' on the server can provide good redundancy

* No single point of failure

* NOTE: STP is required in the subnet in order to break the loops created by the two switches (each one with 2 up-links)

\section{Direct router connections}

is Linux bonding in "active-backup": primary link connected to R1, back-up one to R2

is Not enough:

* example: failure of sw1 primary up-link renders the servers unreachable for Rack1

\section{is Use VLAN interfaces on the routers and interconnect them (emulate a rack} level switch)

* the already existing high speed trunk used by OSPF can be shared by virtually any number of VLANs (tagged)

- Production experience

is Deployed for:

* all (most) critical servers

* the NetApp FAS3100 storage units used system wide (user accounts, etc)

is Sample failure while running:

* a server interface going down and then re-negotiation to a lower speed

* no effect perceived on the data taking run

* Shifter reported the warnings generated by the network monitoring tools

May 2010

IEEE-NPSS Real Time conference, Lisbon 


\section{Control network -high throughput servers}

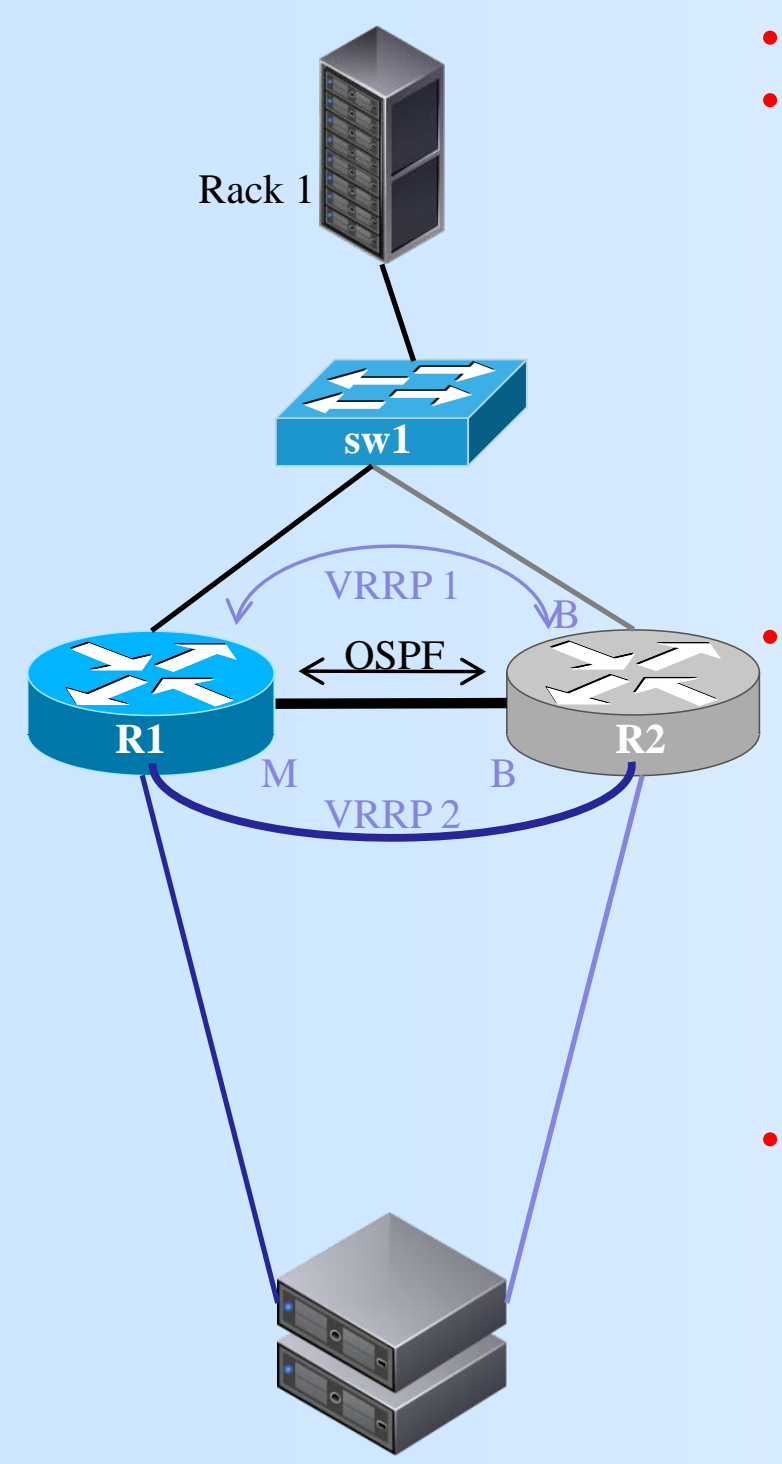

- $\quad$ High throughput needed on $~ 70$ infrastructure and monitoring servers

- "Standard” options:

« Edge switch and VRRP with 10G up-links

* Two points of failure (switch and server interface)

is Edge switch and VRRP with 10G up-links + bonding on the server

* Linux bonding in 'active-backup' on the server can provide good redundancy

* One point of failure (switch)

is Two edge switch and VRRP with 10G up-links + bonding on the server

* Linux bonding in 'active-backup' on the server can provide good redundancy

* No single point of failure

* NOTE: STP is required in the subnet in order to break the loops created by the two switches (each one with 2 up-links)

\section{Direct router connections}

is Linux bonding in "active-backup": primary link connected to R1, back-up one to R2

is Not enough:

* example: failure of sw1 primary up-link renders the servers unreachable for Rack1

\section{is Use VLAN interfaces on the routers and interconnect them (emulate a rack} level switch)

* the already existing high speed trunk used by OSPF can be shared by virtually any number of VLANs (tagged)

- Production experience

is Deployed for:

* all (most) critical servers

* the NetApp FAS3100 storage units used system wide (user accounts, etc)

is Sample failure while running:

* a server interface going down and then re-negotiation to a lower speed

* no effect perceived on the data taking run

* Shifter reported the warnings generated by the network monitoring tools

May 2010

IEEE-NPSS Real Time conference, Lisbon 


\section{Control network -high throughput servers}

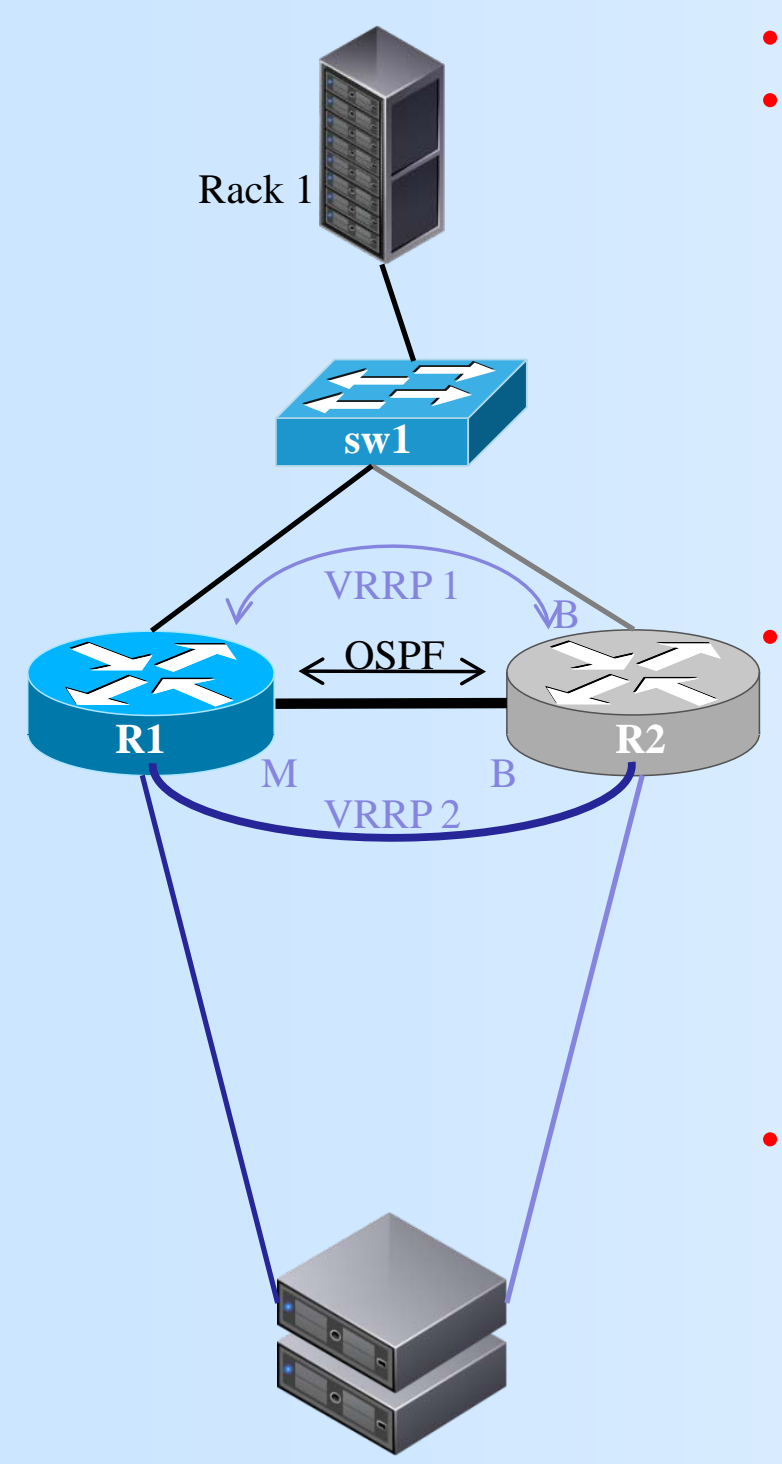

- $\quad$ High throughput needed on $~ 70$ infrastructure and monitoring servers

- "Standard” options:

« Edge switch and VRRP with 10G up-links

* Two points of failure (switch and server interface)

is Edge switch and VRRP with 10G up-links + bonding on the server

* Linux bonding in 'active-backup' on the server can provide good redundancy

* One point of failure (switch)

is Two edge switch and VRRP with 10G up-links + bonding on the server

* Linux bonding in ‘active-backup' on the server can provide good redundancy

* No single point of failure

* NOTE: STP is required in the subnet in order to break the loops created by the two switches (each one with 2 up-links)

\section{Direct router connections}

is Linux bonding in "active-backup": primary link connected to R1, back-up one to R2

is Not enough:

* example: failure of sw1 primary up-link renders the servers unreachable for Rack1

is Use VLAN interfaces on the routers and interconnect them (emulate a rack level switch)

* the already existing high speed trunk used by OSPF can be shared by virtually any number of VLANs (tagged)

- Production experience

is Deployed for:

* all (most) critical servers

* the NetApp FAS3100 storage units used system wide (user accounts, etc)

is Sample failure while running:

* a server interface going down and then re-negotiation to a lower speed

* no effect perceived on the data taking run

* Shifter reported the warnings generated by the network monitoring tools

May 2010

IEEE-NPSS Real Time conference, Lisbon 


\section{BackEnd network}

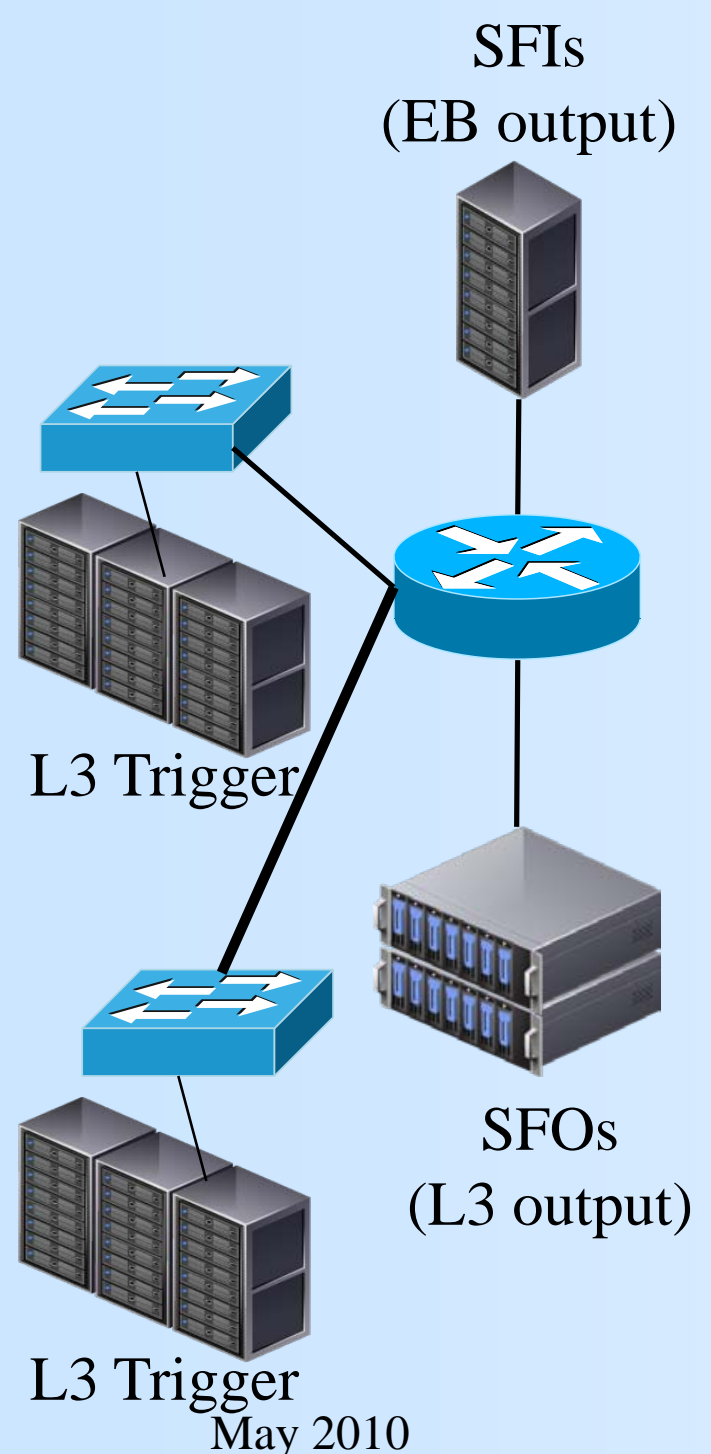

- $\quad$ Routed network (layer 3)

is one subnet for SFIs/SFOs

is one subnet for each Trigger farm

- $\quad$ 802.3ad LAG (Link Aggregation, a.k.a. trunking)

is used on most SFIs, SFOs and a most Trigger farm switches up-links

* dictated by bandwidth requirements

is typically trunks made up of two copper GE ports

* provides built-in redundancy

* if a link goes down (link down) the remaining active ones are transparently used

is on the Core router, LAGs are distributed on multiple line-cards to increase availability

is load balancing

* 802.3ad stipulates order preservation, but this comes at the cost of imperfect load balancing

* Linux bonding and some networking devices offer load balancing modes with "perfect" balancing, at the cost of reordering frames

* Full events ( $>2$ Mbyte) are transferred in the network

$\bigcirc$ Good load balancing (with out-of-order frames) offers better performance for this particular case.

- Production experience

is partial failure of a 12 ports module (each port is a member of a separate LAG - paired with another 12 ports module)

* receive OK, but send corrupted frames

* corresponding links are active so they are used as part of the LAGs

* $~ 50 \%$ frame loss in one direction -> LAGs are unusable

is Provides good redundancy against complete failures, yet vulnerable to incomplete ones. 


\section{Miscellaneous operational issues}

- Link autonegotiation

is Standard (IEEE 802.3ab)

is At power-up, consistently experienced sporadic autonegotiation failures

* links at $10 \mathrm{Mbit} / \mathrm{s}, 100 \mathrm{Mbit} / \mathrm{s}$, or even inconsistent speeds/duplex on the two ends

* Preponderantly on a set of machines with bonded interfaces, but not only

is Various solutions tried out

* No result: cables tested, replaced and re-tested

* Successful: advertise only $1000 \mathrm{Mbit} / \mathrm{s}$ during autonegotiation on the PC side, and try to bring the link up several times in case of failure

practical workaround, but the cause of the problem remains unknown

- Proxy ARP rate limitation

is proxy ARP was initially deployed in the Control network (simplifies end-host management)

is Rare and random connection failures experienced for an information server (receiving data from 1500 clients at the same time)

* problem traced down to be caused by a limitation in the rate of ARPs that can be proxied by the router.

* This was a legitimate DoS protection

is Gave up on using proxy-ARP, and things work smoothly since

- Flooding on ROS data switches

is Message hand-shake between all applications at "prepare for run"

is after 1 h stable beams and the trigger is "turned" on (1000 processors suddently talk to 150 ROSs)

* packet loss is observed on the ROS switches

is Limited rate or "large" latency for re-learning the MAC addresses of the processors

* flooding unnecessarily multiplies the already high rate of frames

* buffer overflows in the corresponding switches, and subsequent warnings (due to late replies)

is Solution: mac-address-aging time increased to a safe value, so that the "prepare for run" message exchange persistently populates the MAC address tables. 


\section{Conclusions}

- Large system with heterogeneous demands (control, Front/Back-End)

- Deployed different flavours of protocols meant at increasing resiliency:

is layer 2: trunking (including bonding on hosts), MST (load balancing)

is layer 3: OSPF, VRRP (no load balancing in current implementation)

- Real life failures are not text-book failures

is very few total failures experienced

is partial failures proved to be more frequent

* since some functionality is preserved, the redundancy protocols may be fooled into using a malfunctioning link.

is foreseeing redundancy reduces down-time, but there is no guarantee that it will always work.

- As the system increased in size, unexpected scaling problems rose to surface (e.g. proxy ARP rate limitation) 